\title{
ISOLATION AND ANTIDIABETIC ACTIVITY OF NEW LANOSTENOIDS FROM THE LEAVES OF PSIDIUM GUAJAVA L.
}

\author{
PRIYANKA BAGRI, MOHAMMED ALI*, VIDHU AERI, MALAY BHOWMIK \\ Faculty of Pharmacy, Jamia Hamdard (Hamdard University), New Delhi 110062, India \\ Email: maliphyto@gmail.com
}

Received: 22 Dec 2015 Revised and Accepted: 22 Jul 2016

\begin{abstract}
Objective: Diabetes mellitus is a chronic metabolic disease which affects our body's ability to use the energy found in food. Our study was planned to isolate chemical constituents from the leaves of Psidium guajava L. (Myrtaceae), to characterize their structures and to investigate their antidiabetic activity.

Methods: The air-dried leaf powder was exhaustively extracted with methanol in a Soxhlet apparatus. The concentrated leaf extact was adsorbed on silica gel (60-120 mesh) for the preparation of a slurry. The dried slurry was chromatographed over silica gel column packed in petroleum ether. The column was eluted with petroleum ether, chloroform and methanol, successively, in order of increasing polarity to isolate the compounds. These natural constituents were tested for the antidiabetic activity in STZ-induced diabetic models.

Results: Six new lanosterol-type triterpenoids characterized as lanost-7-en-3 $\beta$-ol-26-oic acid (2), lanost-7-en-3 $\beta, 12 \beta$-diol-26-oic acid (3), lanost-7-

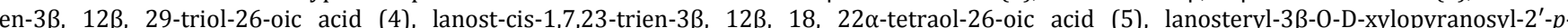

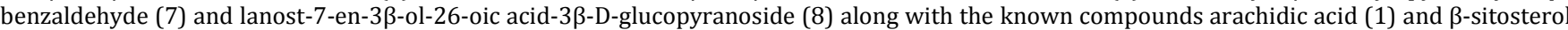
xylopyranoside (6) were isolated from the leaves. The compounds $2,3,4$ and 8 exhibited significant antidiabetic activity against streptozotocin-
\end{abstract} induced diabetic rats.

Conclusion: The leaves of P. guajava possessed antidiabetic lanostene-type triterpenoids.

Keywords: Psidium guajava, Leaves, Lanostenoids, Structural elucidation, Antidiabetic activity

(C) 2016 The Authors. Published by Innovare Academic Sciences Pvt Ltd. This is an open access article under the CC BY license (http://creativecommons. org/licenses/by/4. 0/) DOI: http://dx.doi.org/10.22159/ijpps.2016v8i9.10425

\section{INTRODUCTION}

Diabetes mellitus (DM) is a chronic, lifelong disease which affects our body's ability to use the energy found in food. It is a group of metabolic diseases characterized by high blood sugar, frequent urination, excessive thirst and increased hunger. DM is due to either the pancreas is not producing sufficient insulin or the cells of the body not responding properly to the insulin produced. At present nearly 387 million people, equal to $8.3 \%$ of the adult population with equal rates of males and females, have diabetes worldwide, with type 2 diabetes suffering about $90 \%$ of the cases [1]. Presently, herbal medicines are mainly used to control the disease due to less side effects [2]. In recent years, herbal medicines have started to gain importance as a source of hypoglycemic agents. The plant products are rich in phenolic compounds, flavonoids, terpenoids, coumarins, and other constituents which show a reduction in blood glucose levels $[3,4]$

Psidium guajava L. (Myrtaceae), universally known as guava, is a native to Mexico and Peru and now cultivated in many countries as a shrub or small evergreen tree with many branches $[5,6]$. Its fruits, known as the 'poor man's apple' enrich the diets of millions of people in the tropics of the world [7]. A decoction of the leaves is used as a febrifuge, antispasmodic and to treat bronchitis, asthma, cough, pulmonary diseases and rheumatism. A leaf paste is applied as an antiseptic to cure wounds, ulcers and toothache [8-12]. The guava leaves have been reported to contain $\beta$-sitosterol, pentacyclic triterpenoids, quercetin, avicularin, its 3-L-4-pyranoside, resin, tannin, flavinone-2 2'-ene, prenol, dihydro-benzo-phenanthridine and a volatile oil rich in cineole, eugenol and cryptonine [13-15]. Guavas yield carotenoids and polyphenols, the major classes of antioxidant pigments giving them relatively high potential antioxidant value among plant foods [16]. The leaves showed antimicrobial, antioxidant anticough, central nervous system, hypoglycaemic, antimutagenic and locomotor activities [5, 15]. During the course of our search for bioactive constituents from herbal drugs, we tested a crude extract of $P$. guajava for antidiabetic effect. Based upon the significant antidiabetic activity of the plant extract, we selected $P$. guajava for its phytochemical and antidiabetic studies.

\section{MATERIALS AND METHODS}

General procedures

Melting points were determined by a thermoelectrically heated Perfit melting point apparatus without correction. UV spectra were measured with a Lambda Bio 20 spectrophotometer in methanol Infrared (IR) spectra were recorded using $\mathrm{KBr}$ pellets with a Jasco FT-IR-5000 Spectrometer. The ${ }^{1} \mathrm{H}(400 \mathrm{MHz})$ and ${ }^{13} \mathrm{C}(100 \mathrm{MHz}) \mathrm{NMR}$ spectra were recorded on a Bruker ARX-Spectrometer by using $\mathrm{CDCl}_{3}$ and DMSO- $\mathrm{d}_{6}$ as solvents and TMS as an internal standard. Mass spectrometric detection was carried out on ESI-MS (Q-TOFESI), an electrospray-ionization (ESI) technique with positive ionization mode. Column chromatography was performed on silica gel 60-120 mesh and solvents taken were purchased from Merck Specialities Private Limited. Pre-coated aluminum TLC plates of silica gel 60 F254 were used to run and spots were visualized by exposure to iodine vapors, UV radiations and spraying with an anisaldehyde-sulfuric acid solution.

\section{Plant material}

P. guajava leaves were collected freshly from Faridabad, Haryana, India. The plant was identified by Prof. M. P. Sharma, Taxonomist, Department of Botany, Faculty of Science, Jamia Hamdard, New Delhi. A specimen voucher of the drug was deposited in the Phytochemistry Research Laboratory, Jamia Hamdard with a reference number PRL-JH/2008/05

\section{Extraction and isolation}

The air-dried leaves (1.5 kg) were coarsely powdered and exhaustively extracted with methanol in a Soxhlet apparatus. The combined extracts were concentrated and dried on a steam bath under reduced pressure to get $225 \mathrm{~g}$ of a dark brown mass. It was dissolved in $250 \mathrm{ml}$ methanol and adsorbed on silica gel (60-120 mesh) for the preparation of a slurry. The slurry was dried in air and chromatographed over silica gel column packed in petroleum ether. The column was eluted with petroleum ether, petroleum etherchloroform $(1: 1,1: 3, \mathrm{v} / \mathrm{v})$, chloroform and chloroform-methanol $(97: 3,19: 1$ and $9: 1, \mathrm{v} / \mathrm{v})$ mixtures to isolate the compounds 1-8. 


\section{Arachidic acid (1)}

Elution of the column with petroleum ether furnished colorless crystals of 1 , recrystallized from acetone, $96 \mathrm{mg}(0.064 \%$ yield $)$; $\mathrm{R}_{\mathrm{f}}$ : 0.52 (petroleum ether); m. p. and mixed m. p.: $62-63^{\circ} \mathrm{C}$; +ve FAB MS m/z (rel. int.): $313[\mathrm{M}+\mathrm{H}]^{+}\left(\mathrm{C}_{20} \mathrm{H}_{41} \mathrm{O}_{2}\right)$ (13.1).

\section{Psidiumlanostenoic acid (2)}

Elution of the column with petroleum ether-chloroform (1:1) mixture afforded colorless crystals of 2 , recrystallized from acetone, $886 \mathrm{mg}$ ( $0.059 \%$ yield); Rf: 0.46 (chloroform: methanol; 1:1); m. p.: 176-178 ${ }^{\circ} \mathrm{C}$; UV $\lambda_{\max }(\mathrm{MeOH}): 212,276 \mathrm{~nm}\left(\log \varepsilon\right.$ 5.7, 3.2); IR $v_{\max }$ (KBr): 3415, 3312, 2931, 2850, 1688, 1640, $765 \mathrm{~cm}^{-1} ;{ }^{1} \mathrm{H}$ NMR (DMSO- $\left.d_{6}\right): 5.03(1 \mathrm{H}, \mathrm{brs}, \mathrm{H}-7), 3.75(1 \mathrm{H}, \mathrm{dd}, \mathrm{J}=5.3,8.8 \mathrm{~Hz}, \mathrm{H}-3 \alpha)$ 1.25 (3H, brs, Me-29), 1.22 (3H, brs, Me-28), 1.18 (3H, brs, Me-30), 1.15 (3H,d, J = 6.0 Hz, Me-21), 1.03 (3H, brs, Me-19), 0.92 (3H, d, J = $6.3 \mathrm{~Hz}, \mathrm{Me}-27$ ), 0.85 (3H, brs, Me-18); ${ }^{13} \mathrm{C}$ NMR (DMSO-d 6 ) (table 1);+ve FAB MS $m / z$ (rel. int.): $459[\mathrm{M}+\mathrm{H}]^{+}\left(\mathrm{C}_{30} \mathrm{H}_{51} \mathrm{O}_{3}\right)$ (2.1).

\section{2ß-Hydroxypsidiumlanostenoic acid (3)}

Elution of the column with petroleum ether-chloroform (1:3) mixture yielded colourless crystals of 3 , recrystallized from acetone, $732 \mathrm{mg}$ ( $0.048 \%$ yield); $\mathrm{R}_{\mathrm{f}}$ : 0.48 (chloroform: methanol: 4:1); m. p.: 218-220 ${ }^{\circ} \mathrm{C}$; UV $\lambda_{\max }(\mathrm{MeOH}): 212,277 \mathrm{~nm}(\log \varepsilon 5.3,2.8) ;$ IR $v_{\max }$ (KBr): 3419, 3315, 2932, 2855, 1694, 1640, $989 \mathrm{~cm}^{-1}$; ${ }^{1} \mathrm{H}$ NMR (DMSO- $\left.d_{6}\right): \delta 5.13(1 \mathrm{H}, \mathrm{m}, \mathrm{H}-7), 3.78(1 \mathrm{H}, \mathrm{dd}, \mathrm{J}=5.1,9.5 \mathrm{~Hz}, \mathrm{H}-3 \alpha)$, $3.89(1 \mathrm{H}, \mathrm{dd}, \mathrm{J}=5.2,9.3 \mathrm{~Hz}, \mathrm{H}-12 \alpha), 1.22(3 \mathrm{H}, \mathrm{brs}, \mathrm{Me}-28), 1.20(3 \mathrm{H}$, brs, Me-29), 1.16 (3H, d, J = 6.5 Hz, Me-21), 1.12 (3H, brs, Me-30), 1.04 (3H, brs, Me-19), 0.98 (3H, d, J = 6.0 Hz, Me27), 0.83 (3H, brs, Me-18); ${ }^{13} \mathrm{C}$ NMR (DMSO- $d_{6}$ ) (table 1); FAB MS $m / z$ (rel. int.): 475 $[\mathrm{M}+\mathrm{H}]^{+}\left(\mathrm{C}_{30} \mathrm{H}_{51} \mathrm{O}_{4}\right)$ (2.3).

\section{Trihydroxypsidiumlanostenoic acid (4)}

Elution of the column with chloroform mixture afforded colorless crystals of 4 , recrystallized from acetone, $564 \mathrm{mg}(0.037 \%$ yield); Rf: 0.64 (chloroform: methanol; 4:1); m. p.: $222-225^{\circ} \mathrm{C}$; UV $\lambda_{\max }(\mathrm{MeOH})$ : 210, $281 \mathrm{~nm}\left(\log \varepsilon\right.$ 5.3, 1.1); IR $v_{\max }(\mathrm{KBr}): 3423,3381,3275,2925,1690$, 1630, $956 \mathrm{~cm}^{-1} ;{ }^{1} \mathrm{H}$ NMR (DMSO- $\left.d_{6}\right): \delta 5.13(1 \mathrm{H}, \mathrm{m}, \mathrm{H}-7$ ), 3.75 (1H, dd, J $=5.2,9.3 \mathrm{~Hz}, \mathrm{H}-3 \alpha), 3.86(1 \mathrm{H}, \mathrm{dd}, \mathrm{J}=5.3,9.1 \mathrm{~Hz}, \mathrm{H}-12 \alpha), 3.40(1 \mathrm{H}, \mathrm{brs}$, $\left.\mathrm{HOCH}_{2}-29 \mathrm{a}\right), 3.38$ (1H, brs, $\left.\mathrm{HOCH}_{2}-29 \mathrm{~b}\right), 1.15$ (6H, brs, Me-28, Me-30), 1.04 (3H, brs, Me-19), 0.99 (3H, d, J = 6.6 Hz, Me-21), 0.91 (3H, d, J = 6.1 $\mathrm{Hz}, \mathrm{Me} 27), 0.87$ (3H, brs, Me-18); ${ }^{13} \mathrm{C}$ NMR (DMSO-d $d_{6}$ ) (table 1);+ve FAB MS $m / z$ (rel. int.): $490[\mathrm{M}]^{+}\left(\mathrm{C}_{30} \mathrm{H}_{50} \mathrm{O}_{5}\right)$ (2.6).

\section{Guavalanostenoic acid (5)}

Elution of the column with chloroform-methanol (97:3) mixture furnished colourless crystals of 5, recrystallized from acetone, 462 mg (0.030 \% yield); Rf: 0.70 (chloroform: methanol; 1:1); m. p.: 188$190{ }^{\circ} \mathrm{C}$; UV $\lambda_{\max }(\mathrm{MeOH}): 211,298 \mathrm{~nm}(\log \varepsilon 5.1,2.9) ; \mathrm{IR} v_{\max }(\mathrm{KBr}):$ $3410,3367,3281,2934,2850,1692,1603,990 \mathrm{~cm}^{-1} ;{ }^{1} \mathrm{H}$ NMR (DMSO- $\left.d_{6}\right): \delta 6.51(1 \mathrm{H}, \mathrm{dd}, \mathrm{J}=8.4,9.3 \mathrm{~Hz}, \mathrm{H}-2), 6.79(1 \mathrm{H}, \mathrm{d}, \mathrm{J}=8.4$ $\mathrm{Hz}, \mathrm{H}-1), 5.15(1 \mathrm{H}, \mathrm{d}, \mathrm{J}=9.0 \mathrm{~Hz}, \mathrm{H}-7), 5.12(1 \mathrm{H}, \mathrm{dd}, \mathrm{J}=4.5,8.1 \mathrm{~Hz}, \mathrm{H}-$ 23), $5.01(1 \mathrm{H}, \mathrm{dd}, \mathrm{J}=8.1,5.6 \mathrm{~Hz}, \mathrm{H}-24), 4.49(1 \mathrm{H}, \mathrm{d}, \mathrm{J}=9.3 \mathrm{~Hz}, \mathrm{H}-3 \alpha)$ $4.30(1 \mathrm{H}, \mathrm{dd}, \mathrm{J}=4.5,4.7 \mathrm{~Hz}, \mathrm{H}-22 \beta), 3.89(1 \mathrm{H}, \mathrm{dd}, \mathrm{J}=4.1,11.7 \mathrm{~Hz}, \mathrm{H}-$ $12 \alpha), 3.42\left(1 \mathrm{H}\right.$, brs, $\left.\mathrm{H}_{2}-18 \mathrm{a}\right), 3.39$ (1H, brs, $\left.\mathrm{H}_{2}-18 \mathrm{~b}\right), 1.23$ (3H, brs, Me-29), 1.20 (3H, brs, Me-28), 1.15 (3H, brs, Me-30), 1.02 (3H, d, J = $6.8 \mathrm{~Hz}, \mathrm{Me}-21$ ), 1.09 (3H, brs, Me-19), 0.99 (3H, d, J = $6.2 \mathrm{~Hz}, \mathrm{Me}-27)$; ${ }^{13} \mathrm{C}$ NMR (DMSO- $d_{6}$ ) (table 1);+ve FAB MS $\mathrm{m} / z$ (rel. int.): 503 $[\mathrm{M}+\mathrm{H}]^{+}\left(\mathrm{C}_{30} \mathrm{H}_{47} \mathrm{O}_{6}\right)(2.2)$.

\section{$\beta$-Sitosterol xyloside (6)}

Elution of the column with chloroform-methanol (19:1) mixture gave colourless amorphous powder of 6 , recrystallized from methanol, $286 \mathrm{mg}\left(0.018 \%\right.$ yield), $\mathrm{R}_{\mathrm{f}:} 0.32$ (chloroform: methanol; 4:1), m. p.: $270-272{ }^{\circ} \mathrm{C}$; +ve FAB MS $m / z$ (rel. int.): $547[\mathrm{M}+\mathrm{H}]^{+}$ $\left(\mathrm{C}_{34} \mathrm{H}_{59} \mathrm{O}_{5}\right)(4.3)$.

\section{Psidium lanosteroside (7)}

Elution of the column with chloroform-methanol (9:1) mixture furnished pale yellow crystals of 7 , recrystallized from methanol, $210 \mathrm{mg}$ ( $0.014 \%$ yield); $\mathrm{R}_{\mathrm{f}}: 0.64$ (toluene: ethyl acetate: formic acid; 5:4:1); m. p.: 58-59 ${ }^{\circ} \mathrm{C}$; UV $\lambda_{\max }(\mathrm{MeOH}): 212,278 \mathrm{~nm}(\log \varepsilon$ 4.9, 4.6);
IR $v_{\max }(\mathrm{KBr}): 3441,3386,2921,2837,1703,1644,950,888 \mathrm{~cm}^{-1}$; ${ }^{1} \mathrm{H}$ NMR (DMSO-d $\left.d_{6}\right): \delta 3.81(1 \mathrm{H}, \mathrm{dd}, \mathrm{J}=5.5,9.0 \mathrm{~Hz}, \mathrm{H}-3 \alpha), 1.27(3 \mathrm{H}$, brs, Me-29), 1.25 (3H, brs, Me-28), 1.20 (3H, d, J = $6.3 \mathrm{~Hz}, \mathrm{Me}-21$ ), 1.16 (3H, brs, Me-30), 1.05 (3H, brs, Me-19), 0.98 (3H, d, J = 6.2 Hz, Me-27), 0.96 (3H, d, J = $6.1 \mathrm{~Hz}, \mathrm{Me}-26), 0.89$ (3H, brs, Me-18), 5.09 $\left(1 \mathrm{H}, \mathrm{d}, \mathrm{J}=7.5 \mathrm{~Hz}, \mathrm{H}-1^{\prime}\right), 4.84(1 \mathrm{H}, \mathrm{dd}, \mathrm{J}=7.5,6.3 \mathrm{~Hz}, \mathrm{H}-2 \mathrm{\prime}), 4.71(1 \mathrm{H}$, m, H-3'), $3.96\left(1 \mathrm{H}, \mathrm{m}, \mathrm{H}-4^{\prime}\right), 3.71\left(2 \mathrm{H}, \mathrm{d}, \mathrm{J}=6.5 \mathrm{~Hz}, \mathrm{H}_{2}-5^{\prime}\right), 7.23(2 \mathrm{H}$, m, H-2", H-6"), 7.18 (2H, m, H-3", H-5"), 10.6 (1H, brs, H-7"); ${ }^{13} \mathrm{C}$ NMR (DMSO- $d_{6}$ ) (table 1);+ve FAB MS $\mathrm{m} / \mathrm{z}$ (rel. int.): 667 $[\mathrm{M}+\mathrm{H}]^{+}\left(\mathrm{C}_{42} \mathrm{H}_{67} \mathrm{O}_{6}\right)(1.1), 429\left(\mathrm{C}_{30} \mathrm{H}_{53} \mathrm{O}\right)(15.3)$.

\section{Psidiumlanostenoic acid glucoside (8)}

Further elution of the column with chloroform-methanol (9:1) mixture gave colourless crystals of 8 , recrystallized from methanol, $586 \mathrm{mg}\left(0.037 \%\right.$ yield); $\mathrm{R}_{\mathrm{f}}$ : 0.86 (chloroform: methanol; 4:1); m. p.: 179-180 ${ }^{\circ} \mathrm{C}$; UV $\lambda_{\max }(\mathrm{MeOH}): 214,248 \mathrm{~nm}\left(\log \varepsilon\right.$ 5.3, 4.6); IR $v_{\max }$ (KBr): 3410, 3389, 3250, 2924, 1692, 1650, $808 \mathrm{~cm}^{-1} ;{ }^{1} \mathrm{H}$ NMR (DMSO- $\left.d_{6}\right): \delta 5.28(1 \mathrm{H}, \mathrm{d}, \mathrm{J}=5.3 \mathrm{~Hz}, \mathrm{H}-7), 3.72(1 \mathrm{H}, \mathrm{dd}, 5.2,9.1 \mathrm{~Hz}$, H-3 $\alpha$ ), 1.28 (3H, brs, Me-28), 1.19 (3H, brs, Me-29), 1.15 (3H, brs, Me-30), 1.06 (3H, brs, Me-19), 1.01 (3H, d, J = 6.3 Hz, Me-21), 0.95 (3H, d, J = 6.2 Hz, Me27), 0.83 (3H, brs, Me-18), 5.08 (1H, d, J = 7.1 $\left.\mathrm{Hz}, \mathrm{H}-1^{\prime}\right), 4.17\left(1 \mathrm{H}, \mathrm{dd}, \mathrm{J}=6.9,7.1 \mathrm{~Hz}, \mathrm{H}-2^{\prime}\right), 3.79\left(1 \mathrm{H}, \mathrm{m}, \mathrm{H}-3^{\prime}\right), 3.51$ (1H, m, H-4'), $4.63\left(1 \mathrm{H}, \mathrm{m}, \mathrm{H}-5^{\prime}\right), 3.10\left(1 \mathrm{H}, \mathrm{t}, \mathrm{J}=6.9 \mathrm{~Hz}, \mathrm{H}_{2}-6\right.$ 'a), 3.07 $\left(1 \mathrm{H}, \mathrm{t}, \mathrm{J}=6.9 \mathrm{~Hz}, \mathrm{H}_{2}-6^{\prime} \mathrm{b}\right) ;{ }^{13} \mathrm{C}$ NMR (DMSO- $d_{6}$ ) (table 1); +ve FAB MS $m / z$ (rel. int.): $621[\mathrm{M}+1]^{+}\left(\mathrm{C}_{36} \mathrm{H}_{61} \mathrm{O}_{8}\right)$ (3.1), 440 (7.3), 395 (26.5).

\section{Antidiabetic activity}

Antidiabetic activity of the compounds was performed by streptozotocin (STZ, Sigma chemicals, Mumbai)-induced diabetic model [30]. Male Albino Wistar rats (150-200 g) were used for this study. They were housed in macrolon cages under standard laboratory conditions $\left(12 \mathrm{~h}\right.$ light $/ 12 \mathrm{~h}$ darkness, $\left.21 \pm 2{ }^{\circ} \mathrm{C}\right)$. The animals were given standard pellets diet (Lipton rat feed, Ltd., Pune) and water ad libitum throughout the experimental period. The experimental study was approved by the Institutional Animal Ethical Committee of Jamia Hamdard, New Delhi. The animals were fasted for $16 \mathrm{~h}$ prior to the induction of diabetes. STZ freshly prepared in citrate buffer (0.1 M, pH 4.5) was administered intraperitoneal (i. p.) at a single dose of $60 \mathrm{mg} / \mathrm{kg}$. Development of diabetes was confirmed by polydipsia, polyuria and by measuring blood glucose concentrations $72 \mathrm{~h}$ after injection of STZ. Rats with blood glucose level of $250 \mathrm{mg} / \mathrm{dl}$ or higher were considered to be diabetic. The rats were randomized into six groups comprising of six animals in each groups as given below. Tested compounds 2, 3, 4 and $8(50 \mathrm{mg} / \mathrm{kg})$ were administered orally in aqueous solution $(3 \% \mathrm{v} / \mathrm{v}$ tween 80 in water) once per day.

Group I: control rats, received tween $80(3 \% \mathrm{v} / \mathrm{v}$ in water, 4 $\mathrm{ml} / \mathrm{kg} /$ day, p. o.) $3 \mathrm{~d}$ after citrate buffer (pH-4.5, $1 \mathrm{ml} / \mathrm{kg}$, i. p.) treatment and continued for $7 \mathrm{~d}$.

Group II: diabetic control rats, received STZ in a single dose (60 $\mathrm{mg} / \mathrm{kg}$, i. p.).

Group III: Compound 2 treated diabetic rats, received compound 2 ( $50 \mathrm{mg} / \mathrm{kg} /$ day, p. o.) $3 \mathrm{~d}$ after STZ treatment and continued for $7 \mathrm{~d}$.

Group IV: Compound 3 treated diabetic rats, received compound 3 (50 mg/kg/day, p. o.) $3 \mathrm{~d}$ after STZ treatment and continued for $7 \mathrm{~d}$.

Group V: Compound 4 treated diabetic rats, received compound 4 ( $50 \mathrm{mg} / \mathrm{kg} /$ day, p. o.) $3 \mathrm{~d}$ after STZ treatment and continued for $7 \mathrm{~d}$.

Group VI: Compound 8 treated diabetic rats, received compound 8 ( $50 \mathrm{mg} / \mathrm{kg} /$ day, p. o.) $3 \mathrm{~d}$ after STZ treatment and continued for $7 \mathrm{~d}$.

Blood glucose was estimated by One Touch Glucometer (Accu-Check Roche, Germany).

\section{Statistical analysis}

Data were expressed as the mean \pm SD For statistical analysis of the data, group means were compared by one-way analysis of variance (ANOVA) followed by Turkey post hoc test for multiple comparisons. $P<0.05$ was considered to be statistically significant. 
Table 1: ${ }^{13} \mathrm{C}$ NMR values of compounds 2-5, 7 and 8 isolated from Psidium guajava leaves

\begin{tabular}{|c|c|c|c|c|c|c|}
\hline \multirow[t]{2}{*}{ Carbon position } & \multicolumn{6}{|c|}{${ }^{13} \mathrm{C}$ NMR values } \\
\hline & 2 & 3 & 4 & 5 & 7 & 8 \\
\hline 1 & 36.36 & 36.37 & 36.33 & 130.17 & 35.12 & 36.33 \\
\hline 2 & 27.01 & 23.86 & 25.75 & 138.26 & 26.54 & 25.75 \\
\hline 3 & 79.87 & 80.30 & 78.54 & 83.61 & 77.85 & 78.54 \\
\hline 4 & 39.23 & 39.49 & 39.50 & 38.52 & 39.78 & 39.50 \\
\hline 5 & 46.06 & 41.73 & 42.50 & 46.86 & 56.54 & 42.50 \\
\hline 6 & 18.04 & 17.21 & 17.44 & 18.08 & 19.49 & 17.44 \\
\hline 7 & 124.62 & 124.56 & 124.53 & 121.48 & 28.88 & 124.53 \\
\hline 8 & 138.21 & 138.27 & 138.28 & 143.92 & 44.45 & 138.28 \\
\hline 9 & 54.83 & 54.82 & 52.38 & 54.78 & 50.87 & 52.38 \\
\hline 10 & 38.50 & 38.54 & 38.48 & 37.61 & 36.48 & 38.48 \\
\hline 11 & 22.89 & 21.15 & 22.98 & 23.01 & 24.32 & 22.98 \\
\hline 12 & 30.22 & 67.22 & 67.44 & 79.07 & 25.04 & 67.44 \\
\hline 13 & 41.66 & 46.86 & 46.01 & 47.05 & 26.89 & 46.01 \\
\hline 14 & 47.06 & 47.05 & 46.96 & 50.21 & 47.78 & 46.96 \\
\hline 15 & 33.36 & 33.40 & 33.34 & 33.35 & 31.34 & 33.34 \\
\hline 16 & 32.13 & 32.16 & 33.18 & 32.13 & 32.24 & 33.18 \\
\hline 17 & 52.41 & 52.41 & 52.38 & 52.39 & 50.38 & 52.38 \\
\hline 18 & 15.26 & 16.49 & 13.79 & 64.80 & 15.65 & 13.79 \\
\hline 19 & 17.03 & 17.07 & 17.01 & 17.06 & 20.44 & 17.01 \\
\hline 20 & 36.55 & 37.62 & 37.31 & 36.35 & 36.44 & 37.31 \\
\hline 21 & 21.11 & 18.10 & 21.11 & 21.13 & 21.88 & 21.11 \\
\hline 22 & 32.75 & 32.70 & 32.85 & 73.19 & 39.19 & 32.85 \\
\hline 23 & 27.57 & 27.55 & 27.50 & 125.25 & 24.29 & 27.50 \\
\hline 24 & 31.31 & 31.05 & 30.29 & 115.81 & 42.67 & 30.29 \\
\hline 25 & 29.06 & 30.26 & 30.20 & 30.23 & 25.13 & 30.20 \\
\hline 26 & 178.33 & 178.34 & 178.33 & 178.63 & 19.73 & 178.33 \\
\hline 27 & 23.30 & 23.01 & 23.34 & 23.34 & 19.41 & 23.34 \\
\hline 28 & 23.84 & 23.33 & 23.81 & 23.83 & 28.86 & 23.81 \\
\hline 29 & 28.26 & 28.87 & 63.91 & 28.85 & 16.64 & 27.25 \\
\hline 30 & 16.10 & 16.96 & 17.02 & 16.47 & 16.67 & 15.58 \\
\hline $1^{\prime}$ & -- & -- & -- & -- & 106.32 & 101.53 \\
\hline $2^{\prime}$ & --- & --- & --- & --- & 73.52 & 71.95 \\
\hline $3^{\prime}$ & --- & --- & --- & --- & 68.49 & 67.17 \\
\hline $4^{\prime}$ & --- & --- & --- & --- & 67.16 & 66.01 \\
\hline $5^{\prime}$ & --- & --- & --- & -- & 61.23 & 76.53 \\
\hline $6^{\prime}$ & --- & --- & --- & --- & -- & 62.99 \\
\hline $1^{\prime \prime}$ & --- & --- & --- & --- & 168.41 & --- \\
\hline $2^{\prime \prime}$ & --- & --- & --- & --- & 127.40 & --- \\
\hline $3^{\prime \prime}$ & --- & --- & --- & --- & 125.51 & --- \\
\hline $4^{\prime \prime}$ & --- & --- & --- & --- & 130.06 & --- \\
\hline $5^{\prime \prime}$ & --- & --- & --- & --- & 124.07 & --- \\
\hline $6^{\prime \prime}$ & --- & --- & --- & --- & 125.84 & -- \\
\hline
\end{tabular}

\section{RESULTS}

Elution of the column with petroleum ether gave arachidic acid (1). The compounds 2 and 3 were obtained in petroleum etherchloroform mixtures. Further elution of the column with chloroform and methanol mixtures afforded compounds from 4 to 8 . All the phytoconstituents were obtained in crystalline forms. Diabetes was induced in rats by intraperitoneal injection of STZ solution. Aqueous solutions of the tested compounds were administered orally once a day.

\section{DISCUSSION}

Compounds 1 and 6 are the known compounds characterized as arachidic acid and $\beta$-sitosterol xylopyranoside [17].

Compounds 2-8 responded positively to Liebermann-Burchard test and yielded effervescences with sodium bicarbonate solution indicating triterpenic acid nature of the molecules. Their IR spectra displayed characteristic absorption bands for hydroxyl functions (3410-3275 $\left.\mathrm{cm}^{-1}\right)$, carboxylic groups $\left(1692-1688 \mathrm{~cm}^{-1}\right)$ and unsaturation (1603-1650 $\left.\mathrm{cm}^{-1}\right)$. The molecular ion peaks were determined on the basis of mass and ${ }^{13} \mathrm{C}$ NMR spectra and the position of vinylic bonds and functional groups were established on the basis of mass ion fragments. The ${ }^{1} \mathrm{H}$ NMR spectra of the compounds displayed signals for vinylic protons from $\delta 6.79$ to 5.03, oxygenated $\mathrm{H}-3 \alpha$ methine protons from $\delta$ 4.49-3.72 and methyl protons between $\delta 1.22-0.83$, all attached to the saturated carbons.
The ${ }^{13} \mathrm{C}$ NMR spectra of the compounds exhibited signals for vinylic carbons from $\delta 143.92$ to 115.81 , carboxyl carbons near $\delta 178.33$, C3 carbinol or oxygenated methine carbons between $\delta$ 83.61-77.85 and methyl carbons in the upfield region from $\delta 28.87$ to 13.79 . The ${ }^{13} \mathrm{C}$ NMR spectrum of 7 displayed C-3 carbinol signal at $\delta 77.85$, anomeric carbon at $\delta 106.32\left(\mathrm{C}-1^{\prime}\right)$, aromatic signals at 168.41 (C$\left.1^{\prime \prime}\right), 127.40$ (C-2"), 125.51 (C-3"), 130.06 (C-4"), 124.07 (C-5"), $12.84\left(\mathrm{C}-6^{\prime \prime}\right)$, sugar carbons between $\delta 73.52-67.16$ and aldehydic C$7^{\prime \prime}$ carbon at $\delta 192.19$. The ${ }^{13} \mathrm{C}$ NMR spectrum of 8 also exhibited signals for anomeric carbon at $\delta 101.53\left(\mathrm{C}-1^{\prime}\right)$ and other sugar carbons resonated between $\delta$ 76.53-62.99. The ${ }^{1} \mathrm{H}$ and ${ }^{13} \mathrm{C}$ NMR spectral data of the isolated compounds were compared with the lanosterol-type triterpenoids [18-20]. The ${ }^{1} \mathrm{H}-{ }^{1} \mathrm{H}$ COSY spectra of the triterpenoids showed correlations of the adjacent protons. The HMBC spectra of these compounds exhibited interactions of protons with the adjacent carbons. On the basis of spectral data analyses and chemical reactions, the structures of the isolated phytoconstituents

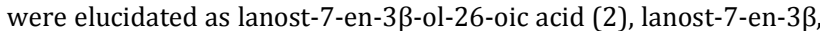

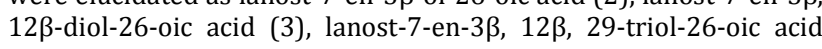
(4), lanost-cis-1,7,23-trien-3 $\beta, 12 \beta, 18,22 \alpha$-tetraol-26-oic acid (5), lanosteryl-3 $\beta$-0-D-xylopyranosyl-2'- $p$-benzaldehyde (7) and lanost7-en-3 $\beta$-ol-26-oic acid-3 $\beta$-D-glucopyranoside (8).

Compound 2, 3, 4 and 8 were tested for the antidiabetic activity in STZ-induced diabetic models [21]. Table 1 shows the levels of blood glucose level in normal and experimental animals in each group. 


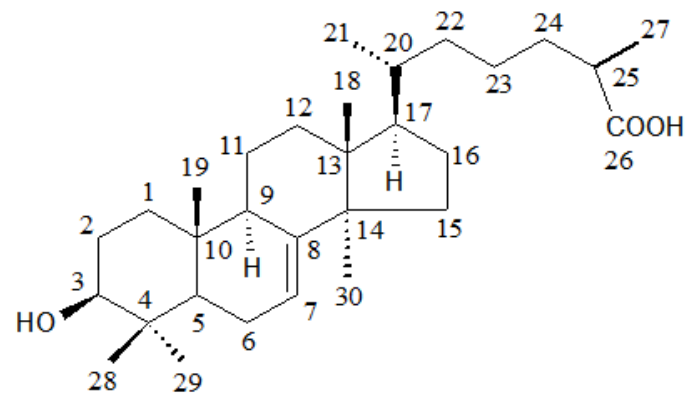

(2)

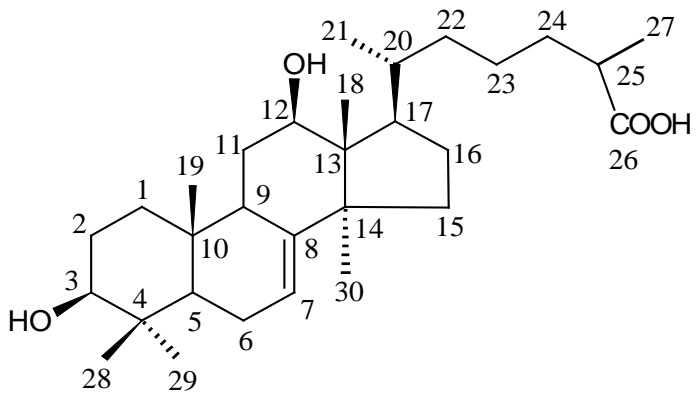

(3)

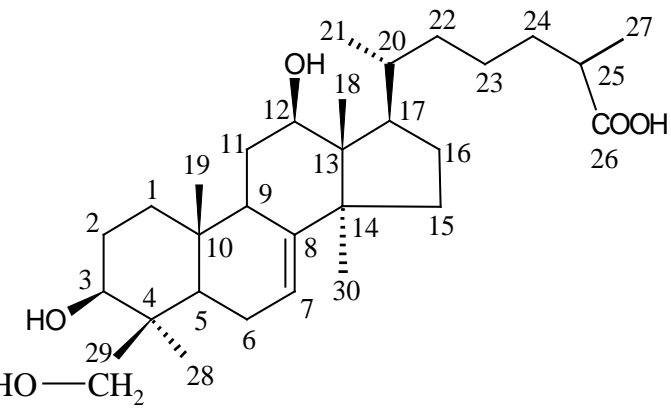

(4)

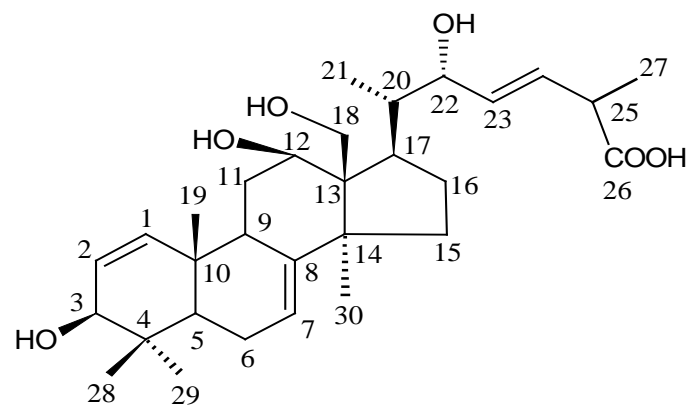

(5)

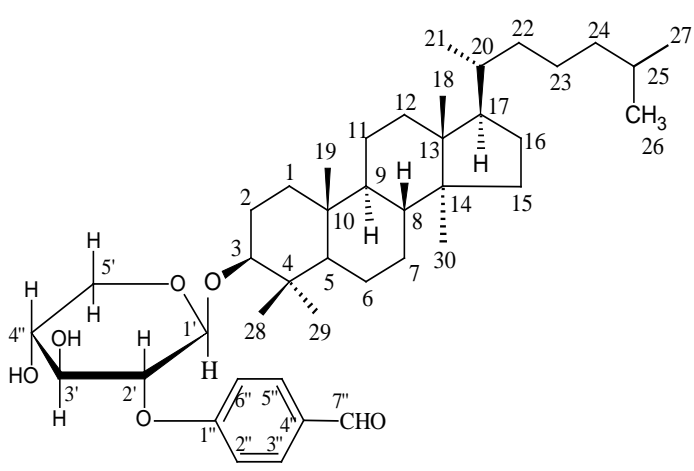

(7)

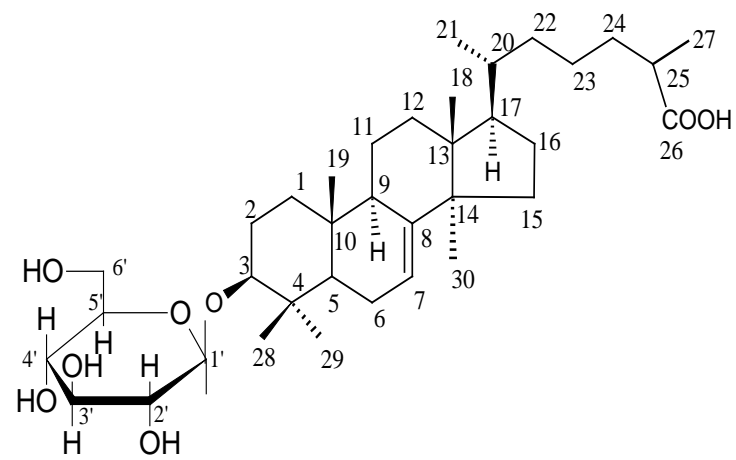

(8)

Fig. 1: Structural formulae of compounds 2-5, 7 and 8

The isolated phytoconstituents have been found to show significant antidiabetic activity. In all the groups prior to STZ administration, the basal levels of blood glucose of the rats were not significantly different.

However, after STZ administration, significant $(P<0.001)$ elevation in blood glucose level was observed in diabetic control rats (group II) when compared with normal control rats (group I). Oral administration of the selected isolated compounds at a dose of 50 $\mathrm{mg} / \mathrm{kg}$ for one week significantly $(P<0.001)$ reduced the blood glucose levels in diabetic rats as compared with diabetic control rats (table 2). This implies that the compounds can prevent or be helpful in controlling blood glucose level near to normal levels, a key for preventing or reversing diabetic complications. In conclusion, the present study demonstrates that the isolated compounds from the leaves of $P$. guajava at tested dose level exhibit potent antidiabetic potential in STZ-induced diabetic rats.

Further studies are warranted in this area to outline precise mechanism behind the antihyperglycemic property of these compounds.

Table 2: Effect of isolated compounds on blood glucose level in diabetic rats

\begin{tabular}{llll}
\hline Groups & Blood glucose $\mathbf{( m g ~ d l}-\mathbf{-})$ & & First week \\
\cline { 2 - 4 } & $\mathbf{0 ~ d}$ & $\mathbf{3 ~ d ~ a f t e r ~ S T Z}$ & $87.66 \pm 8.80$ \\
\hline Normal control & $90.34 \pm 8.89$ & -- & $327.33 \pm 6.41^{\#}$ \\
Diabetic control & $83.66 \pm 11.62$ & $311.50 \pm 8.24$ & $172.17 \pm 6.41^{* *}$ \\
Diabetic+compound $2(50 \mathrm{mg} / \mathrm{kg})$ & $100.67 \pm 10.71$ & $309.50 \pm 8.57$ & $154.50 \pm 6.06^{* *}$ \\
Diabetic+compound $3(50 \mathrm{mg} / \mathrm{kg})$ & $87 \pm 11.78$ & $310.16 \pm 8.86$ & $142.50 \pm 12.03^{* *}$ \\
Diabetic+compound $4(50 \mathrm{mg} / \mathrm{kg})$ & $84.50 \pm 12.42$ & $315.50 \pm 6.38$ & $165.67 \pm 8.73^{* *}$ \\
Diabetic+compound $8(50 \mathrm{mg} / \mathrm{kg})$ & $90.33 \pm 12.88$ & $325.66 \pm 8.71$ & \\
\hline
\end{tabular}

The data are expressed in mean $\pm \mathrm{SD} ; \mathrm{n}=6$ in each group. ${ }^{\#} P<0.001$ compared with the corresponding values for glibenclamide [22] treated animals. 


\section{CONCLUSION}

Six new lanosterol-type triterpenoids, arachidic acid, and $\beta$-sitosterol xylopyranoside were isolated from the leaves of Psidium guajava for the first time. The triterpenic constituents exhibited significant antidiabetic activity against streptozotocin-induced diabetic rats.

\section{ACKNOWLEDGEMENT}

The authors express their gratitude to the Head, SAIF, Central Drug Research Institute, Lucknow for recording mass spectra of the compounds.

\section{CONFLICTS OF INTERESTS}

The authors declare no conflict of interest.

\section{REFERENCES}

1. Joshi SK, Shrestha S. Diabetes mellitus a review of its associations with different environmental factors. Kathmandu University Med J 2010;8:109-15.

2. Patel K, Srinivasan K. Plant foods in the management of diabetes mellitus: vegetables as potential hypoglycemic agents. Nahrung 1997;41:68-74.

3. Marles RJ, Farnsworth NR. Antidiabetic plants and their active constituents. Phytomedicine 1995;2:137-89.

4. Jung M, Park M, Lee HC, Kang Y, Kang ES, Kim SK. Antidiabetic agents from medicinal plants. Curr Med Chem 2006;13:1203-18.

5. Shruthi SD, Roshan A, Sharma S, Timilsina SS, Sunita S. A review on the medicinal plant Psidium guajava Linn. (Myrtaceae). J Drug Delivery Ther 2013;3:162-8.

6. Lucky O Okunrobo, Kate E Imafidon, Adeyemi A Alabi. Phytochemical, proximate and metal content analysis of the leaves of Psidium guajava Linn (Myrtaceae). Int J Health Res 2010;3:217-21.

7. El-Mahmood MA. The use of Psidium guajava Linn. in treating wound, skin and soft tissue infections. Sci Res Essay 2009;4:605-11.

8. Anonymous. The Wealth of India. A Dictionary of Indian Raw material and Industrial products, National Institute of Science Communication and information Resources, CSIR, New Delhi; 2003. p. 286-93.

9. Leonti M, Vibrans H, Stiche O, Heinrich M. Ethnopharmacology of the popoluca, mexico: an evaluation. J Pharm Pharmacol 2001;53:1653-69.
10. Batick MJ. Ethnobotany of palms in the neotropics. In: Prance GT, Kallunki JA. editors. Advances in Economic Botany: Ethnobotany in the Neotropics. New York, USA: New York Botanical Garden; 1984. p. 9-23.

11. Khan MLH, Ahmad J. A pharmacognostic study of Psidium guajava L. Int J Crude Drug Res 1985;23:95-103.

12. Nadkarni KM, Nadkarni AK. Indian Materia Medica-with Ayurvedic, Unani-Tibbi, Siddha, Allopathic, Homeopathic, Naturopathic and Home remedies. Popular Prakashan Private Ltd., Bombay, India; 1991. p. 142-9.

13. Begum S, Hassan SI, Ali SN, Siddiqui BS. Chemical constituents from the leaves of Psidium guajava. Nat Prod Res 2004;18:135-40.

14. Latza S, Ganber D, Berger RG. Carbohydrate esters of cinnamic acid from fruits of Physalis peruviana, Psidium guajava and Vaccinium vitis-idaea. Phytochemistry 1996;43:481-5.

15. Matsuo NH, Koyoko SY, Nakamura, Tomita I. Identification of $(+)$ gallocatechin as a bio-antimutagenic compound in Psidium guajava leaves. Phytochemistry 1994:36:1027-9.

16. Jimenez-Escrig M, Rincon M, Pulido R, Saura-Calixto F. Guava fruit (Psidium guajava L.) as a new source of antioxidant dietary fiber. J Agric Food Chem 2001;49:5489-93.

17. Alam S, Ali M, Alam P, Shuaib M. Phytochemical investigation of the seeds of Butea monosperma. Chem Nat Compds 2010;46:44-8.

18. Ali M. Techniques in terpenoid identification, Birla Publications. Delhi; 2001. p. 352-60.

19. Khan MA, Ali M, Alam P. Phytochemical investigation of the fruit peels of Citrus reticulata Blanco. Nat Prod Res 2010;24:610-20.

20. Chung IM, Ali M, Yang YM, Peebles CAM, Chun SC, Lee SJ, et al. Identification of new compounds from Catharanthus roseus hairy root culture. Bull Korean Chem Soc 2007;28:1294-8.

21. Szkudelski T. The mechanism of alloxan and streptozotocin action in B cells of the rat pancreas. Physiol Res 2001;50:537-46.

22. Upadhyay P, Pandit JK, Sukirti Upadhyay S, Ghosh AK. Studies on formulation and optimization of gastro retentive multiparticulates of glibenclamide and metformin hydrochloride for the treatment of type II diabetes mellitus using gelucire: a review. J Pharm Sci Res 2010;2:351-4.

\section{How to cite this article}

- $\quad$ Priyanka Bagri, Mohammed Ali, Vidhu Aeri, Malay Bhowmik. Isolation and antidiabetic activity of new lanostenoids from the leaves of Psidium guajava $\mathrm{l}$. Int J Pharm Pharm Sci 2016;8(9):14-18. 